\title{
Advances in pediatric diffusion tensor imaging
}

\author{
Robert C. McKinstry
}

(C) Springer-Verlag 2011

Diffusion imaging has a firmly established role in neuroimaging of the child. Diffusion-weighted images (DWI) and apparent diffusion coefficient maps (ADC) (a.k.a. mean diffusivity or MD) have been staples in modern brain imaging protocols for most of a decade. DWI and ADC images can be computed from acquisitions using three orthogonal diffusionencoding gradient orientations, which yield so-called traceweighted images. These images are rotationally invariant where the DWI contrast and ADC values are independent of head position or rotation within the scanner. DWI and ADC have seen widespread clinical application in pediatrics including characterization of newborn brain injury, metabolic disease and brain tumor cellularity.

Diffusion tensor imaging (DTI) relies on acquisition of at least six sets of images using non-collinear diffusionencoding gradient orientations. The model assumed by the diffusion tensor is that diffusion in tissue is an ellipsoid described by three orthogonal diffusion coefficients (eigenvalues) and three rotation angles (eigenvectors) that describe the directional preference of diffusion relative to the $\mathrm{x}, \mathrm{y}$, and $\mathrm{z}$ coordinates of the MR scanner. Rotationally invariant DWI and ADC images are easily computed from the diffusion tensor by taking the mathematical trace of the diffusion tensor. Anisotropy is a measure of how different each of the six elements of the diffusion tensor is from the others. This can be expressed as a normalized standard deviation or coefficient of variation. The most commonly

Disclaimer Dr. McKinstry has no financial interests, investigational or off-label uses to disclose.

R. C. McKinstry $(\bowtie)$

Washington University School of Medicine,

St. Louis, MO 63110, USA

e-mail: McKinstryb@mir.wustl.edu used metric for anisotropy is fractional anisotropy (FA). FA approaches zero when diffusion is the same in all directions (e.g., fluid) and it approaches one when the voxel is filled with tightly packed, highly organized white matter fibers (e.g., splenium of the corpus callosum). FA has the benefit of being rotationally invariant as well. Most vendors now also supply a DTI reconstruction that also produces a directionally encoded color map (DEC). Color is assigned based on white matter fiber orientation where red is left/right, green is anterior/posterior and blue is through plane on an axial image. Commonly, the color information is superimposed onto the FA map so that one perceives the magnitude the anisotropy and the direction the fibers travel simultaneously. This aids in the identification of, or the displacement or disruption of, specific white matter fiber tracts. It should be noted that DEC is not rotationally invariant, as the color scheme is usually defined by the magnet $(\mathrm{x}, \mathrm{y}$, and $\mathrm{z}$ ) coordinates. As a result, the color assignment could change if the head were rotated in the field of view.

DTI metrics (ADC and FA) have been used to quantify brain development and maturation by a number of investigators. ADC falls rapidly during the first few years of life, likely representing decreasing free-water content. Conversely, FA increases in white matter as the phases of myelination proceed and the white matter achieves a more mature form. DTI has been incorporated into a number of pediatric multicenter studies including the NIH MRI Study of Normal Brain Development (http://nih-pediatricmri.org) and the Infant Brain Imaging Study (http://www.ibis-network.org), the later studying brain development in autism.

DTI is also being incorporated into studies of prematurity to examine the impact of very-low birth weight by comparing premature newborns at term-adjusted gestational age and comparing them with term control newborns. Because of the quantitative nature of DTI, investigators are 
able to test therapeutic interventions as well as changes in management in the neonatal intensive care unit to help achieve optimal short-term imaging outcomes. Ultimately, long-term follow-up and clinical outcomes will be needed to ensure that good imaging outcomes predict good neurodevelopmental outcomes.

DTI has been a useful addition to the evaluation of brain injury in term newborns as well. Studies have addressed the time course of ADC changes following the injury and the distribution of the ADC abnormalities with length and severity of the insult. Diffusion also has been shown to highlight early signs of Wallerian degeneration and alterations in the posterior limb of the internal capsule, which many investigators believe imports significant prognostic significance. Early reports and ongoing studies investigate how therapeutic interventions such as cooling alter the distribution and time course of ADC changes following brain injury, and how that translates to patient outcomes.

As mentioned previously, DTI characterizes the orientation of the white matter tracts by solving for the angle the tract follows (eigenvector) relative to the MR scanner. Using a number of mathematical approaches, it is possible to construct white matter tract maps. In the clinical setting,
DTI tractography is most useful for informing the neurosurgeon of the proximity of key white matter tracts relative to a planned resection for epilepsy or tumor. In the lab, DTI tractography is being investigated as a metric for quantifying normal white matter tract development and for comparing tract organization between normal and disease states (e.g., dyslexia, autism and hydrocephalus).

Although DTI has great potential for improving our understanding of the developing brain and childhood neurological disease, it is not a perfect tool. The diffusion ellipsoid that is described by the diffusion tensor is sufficient only for a single bundle of white matter fibers traveling in one direction. To fully describe diffusion in a more realistic setting where white matter fibers are crossing and branching, six diffusion-encoding directions and routine DTI metrics are insufficient. The field is shifting focus to high-angular-resolution diffusion imaging (HARDI) and advanced mathematical formalisms to improve our ability to understand diffusion in a more complex but realistic model of white matter organization in the brain. The resultant images dramatically illustrate the shortcomings of DTI and the potential for HARDI to help us understand the developing human connectome in health and disease. 